\title{
A NEW MODULAR THREE-PHASE INVERTER BASED ON SEPIC-CUK COMBINATION CONVERTER FOR PHOTOVOLTAIC APPLICATIONS
}

\author{
Saud Alotaibi ${ }^{*}$, Ahmed Darwish ${ }^{1}$, Xiandong Ma $^{1}$ and Barry W. Williams ${ }^{2}$ \\ ${ }^{1}$ Lancaster University, Department of Engineering, Lancaster, United Kingdom \\ ${ }^{2}$ University of Strathclyde, Electrical and Electronic Engineering Department, Glasgow, United Kingdom \\ *s.alotaibi@lancaster.ac.uk
}

Keywords: Modular DC/AC Inverter, SEPIC converter, Cuk converter, Photovoltaic applications.

\begin{abstract}
A new modular dc/ac inverter based on a dual-winding isolated SEPIC/Cuk converter for medium and high power Photovoltaic (PV) applications is introduced. In this system, several current-source submodules (SMs) are connected in series to allow for additional voltage boosting. Each SM is designed as a combination of SEPIC/CUK converter to offer a flexible output range and continuous currents with small ripple at input and output sides. Furthermore, the SMs structure can generate different output voltage polarities. The main purpose of employing small-size highfrequency transformers are to (1) provide galvanic isolation, (2) eliminate PV grounding problems, (3) achieve minimum electromagnetic interference (EMI). The paper presents a description of the proposed topology and investigate its reliability performance in a PV grid-tied system using MATLAB simulations. An experimental prototype has been used and controlled by TMS32028335 DSP, as a proof of concept.
\end{abstract}

\section{Introduction}

With the present high global energy consumption and industrial progress, fossil fuels are still considered as primary energy sources to meet the energy demand requirements. Consequently, climate change and green gas emissions have been existing global challenges. As the conventional energy sources (i.e. fossil fuels) have limitations of the product life cycle for the coming years, the global energy demands will be difficult to satisfy [1].

Alternatively, Renewable Energy Sources (RESs) are now considered for a weighty portion of energy generation and as a possible technology to solve the limited quantity of fossil fuels. Furthermore, RESs are anticipated to contribute significantly to future energy efficiency, security, and reduction in $\mathrm{CO} 2$ emissions [1].

Among all types of RES, the installed capacity of solar photovoltaic (PV) generators is witnessing the fastest growth and the total solar energy production was expected to exceed $500 \mathrm{GW}$ in 2018. This rapid increase in the PV systems' capacity is spearheaded by European countries where PV capacity reached $103 \mathrm{GW}$ in 2016. Therefore, the presence of photovoltaic systems (PV) becomes inevitable in the power generation area [2].
Traditionally, power converters for grid-connected photovoltaic systems (PV) particularly Medium and large-scale power systems face fundamental challenges. For example, it is required to install a bulky step-up transformer to meet the essential grid voltage boosting. The use of this transformer introduces high installation and maintenance costs in energy conversion systems $[3,4]$. Furthermore, it is known that the absence of isolation between the PV system and ground (as with the grid) is responsible for creating of common-mode voltages, which can cause leakage currents to flow through the PV system. Consequently, dangerous current levels may arise and hence affect the operation, safety and lifetime of a PV system negatively $[5,6]$.

The significant progress of solid-state semiconductor devices in the last few decades has led to considerable improvement in grid-connected photovoltaic systems (PV). The modern power electronic topologies have developed to open a new horizon for enhancing switching efficiency, design optimisation and reducing the total power losses [7]. Therefore, the employed power inverters in PV applications should be able to offer bidirectional power flow and satisfying other requirements such as tracking the maximum power point as well as reducing the size, weight, and cost $[8,9]$. This will allow for mitigating PV system problems and for 
achieving efficient operations under various frequencies and weather conditions [10].

In this paper, a new modular inverter topology for gridconnected photovoltaic systems (PV) based on the dualwinding isolated SEPIC/Cuk converter is proposed. The inverter modular structure provides high-quality output voltages and currents into the grid without using large transformer components. Furthermore, it improves system's reliability, especially during dc and ac faults. If some submodules are faulted for any unexpected reason, they can be by passed or replaced with redundant submodules.

The most interesting feature in the inverter structure is its ability for employing high-frequency transformers which can offer the required galvanic isolation. The presence of the isolation is important for solving the grounding issues of the PV panels and improve the safety concerns of the system. Moreover, with increasing the operating frequency of the inverter, the sizes of these isolation transformers can be reduced significantly.

Because the employed submodules are based on the SEPIC-Cuk converters, the proposed inverter has the advantage of providing smooth continuous input currents that are desirable for the MPPT controller operational. Thus, recourse to plastic or film capacitor filtering instead of employing a bulky electrolytic capacitor, which is the main source of unreliability in the PV system.

The rest of this paper gives a detailed description of the proposed inverter system in section 2, along with the modulation technique which will be discussed in section 3. Then, the principle of operation of this system will be presented in section 4 . The inverter system has been evaluated with simulations, using MATLAB/Simulink and the results are described. Finally, a scaled-down prototype is developed and controlled with TMS32028335 DSP.

\section{SYSTEM DESCRIPTION}

Fig.1 shows a novel architecture of the three-phase modular structure which is formed by the series connection of submodules (SMs) consisting of isolated SEPIC/Cuk converters. Each SM has an identical front structure and two output terminals that are beneficial for performing efficient power conversion by providing dual output voltages. The unique feature here is that the gate signal is the same for the output switches $S_{a N}$ and $S_{b N}$. Therefore, their output voltages have equal magnitude, but opposite polarity. Where the output voltage is positive for the upper terminal (SEPIC output) and negative for the lower terminal (Cuk output).

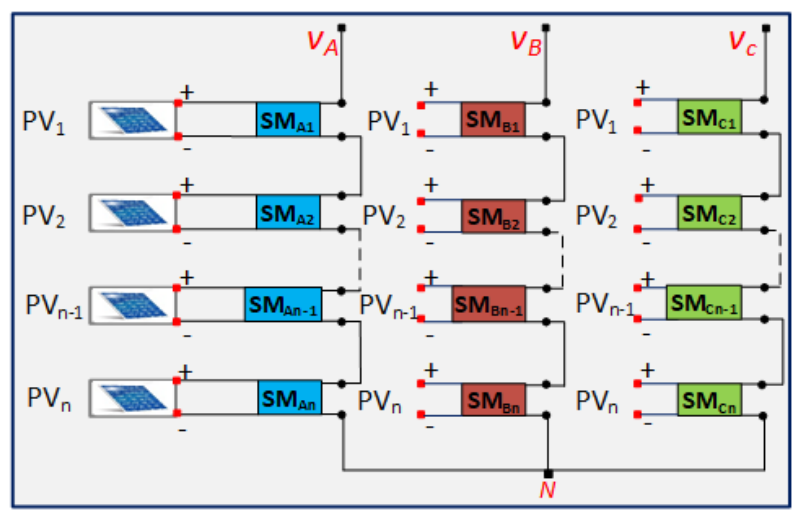

Figure 1 Block diagram of proposed system

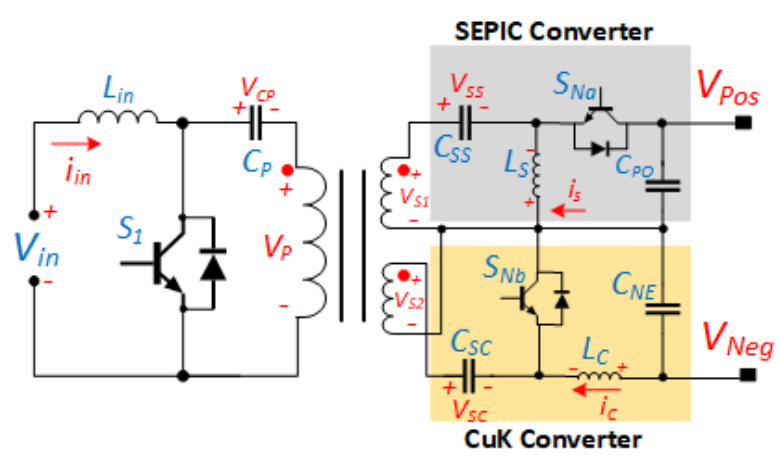

Figure 2 The employed SM

\section{Modulation technique}

To operate the SEPIC/CUK converter, the duty cycle ratio (D) is compared with a saw-tooth carrier signal to generate the PWM signals for the converter's switches. Fig.3 shows the output voltage waveforms of two successive submodules (SMs). Each SM generates a sinusoidal ac voltage plus a dc voltage component. The resultant voltage of the two successive SMs is shown in Fig. 3 as ac voltage at the grid frequency. Each converter SM can be connected individually to a PV module it can be clustered with other SM in parallel with the PV module.

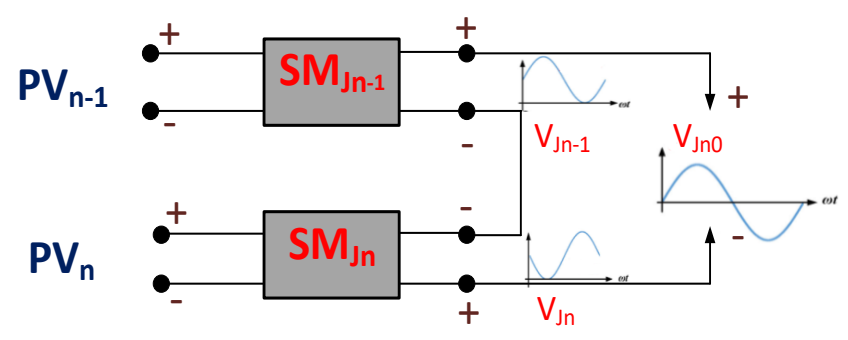

Figure 3 Modulation scheme

Where $\mathrm{J}$ the phases ( $\mathrm{a}, \mathrm{b}$ or $\mathrm{c}$ ) and $\mathrm{n}$ presented number of the modules. Thus, the output voltage for each SM can be delivered as follows. 


$$
\begin{gathered}
V_{J n-1}=\frac{1}{2} V_{n-1} \sin \omega t+V_{d c n-1} \\
V_{J n}=\frac{1}{2} V_{n} \sin (\omega t+\pi)+V_{d c n} \\
V_{J n O}=\frac{1}{2} V_{n-1} \sin \omega t+V_{d c n-1} \\
\quad-\frac{1}{2} V_{n} \sin (\omega t+\pi)-V_{d c n}
\end{gathered}
$$

Since both $V_{J n-1}$ and $V_{J n}$ are equalled alongside $V_{d c_{n-1}}=V_{d c_{n}}$ consequently the out voltage is;

$$
V_{J n O}=V_{n} \sin \omega t
$$

\section{Modes of operation}

The circuit topology is consisting of three switches and diodes. The complementary switching singles of $S_{1}$ and $\mathrm{S}_{\mathrm{N}}$ control the output voltage of the circuit. Both switches $\mathrm{S}_{\mathrm{aN}}$ and $\mathrm{S}_{\mathrm{bN}}$ are turned on and off at the same time. Without losing the generality, explaining the operation can be conducted for non-isolated version in continuous conduction mode (CCM). Thus, system operation is associated with the states of $S_{1}$ and $S_{N}$ switches which can be divided into two operating modes.

Mode 1:

The switch $S_{1}$ turned on and the $S_{N}$ is off, the flowing current paths are shown in Fig.4. In this mode, the input inductor is charged by its current $I_{\text {in }}$ and both SEPIC and Cuk capacitors are connected to ground by the switch $S_{1}$. Hence, $L_{s}$ is energised by the current $I_{s}$. Meanwhile, the SEPIC capacitor $\mathrm{C}_{\mathrm{s}}$ is discharging and its energy is transferred $\mathrm{L}_{\mathrm{s}}$. While the Cuk inductor is charging because the voltage magnitude of input side is greater than outside causes Cuk capacitor to release its energy stored into Cuk inductor.

\section{Mode 2:}

As shown in Fig.5 during $\mathrm{S}_{\mathrm{N}}$ is on and $\mathrm{S}_{1}$ is off, the inductor $\mathrm{L}_{\text {in }}$ transfers its energy to the capacitors $\mathrm{C}_{\mathrm{s}}$ and $\mathrm{C}_{\mathrm{c}}$. Therefore, the input current $\mathrm{I}_{\text {in }}$ decreases. Meanwhile, the output inductors $\mathrm{L}_{\mathrm{s}}$ and $\mathrm{L}_{\mathrm{c}}$ also transfer their energies to $C_{p}$ and $C_{N}$ respectively and the magnitude of $I_{s}$ and $I_{c}$ decrease.

\section{Simulation results}

The inverter system has been built and investigated in open loop operation. The system is simulated using MATLAB/Simulink to study the inverter performance in detail. The model has four SMs per phase and its parameters are given in Table 1.

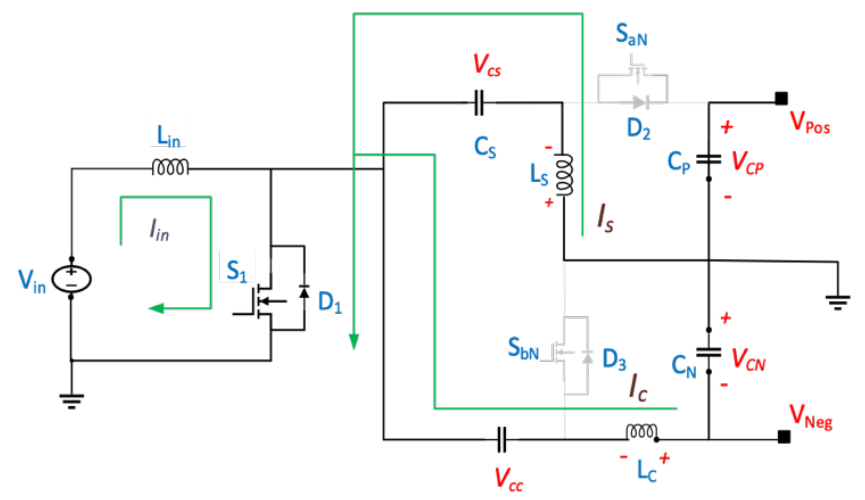

Figure 4 Mode 1

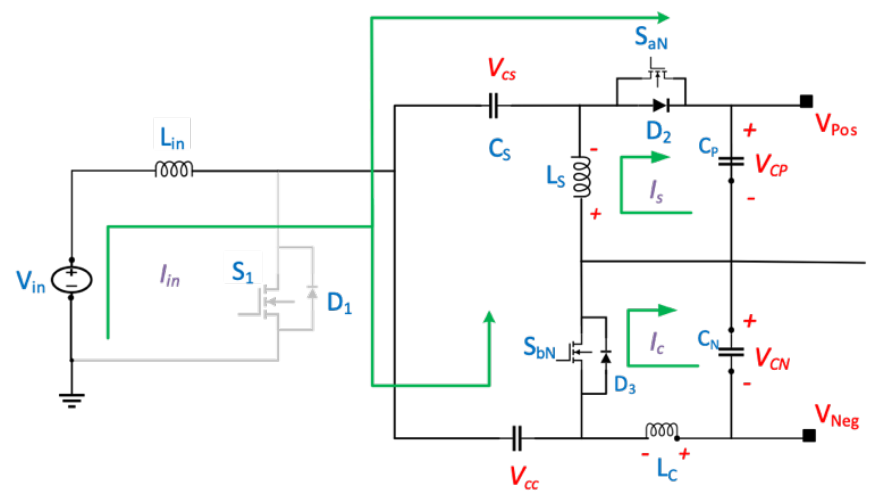

Figure 5 Mode 2

$\begin{array}{ll}\text { Parameters } & \text { Value }\end{array}$

\begin{tabular}{cc}
\hline (SMs) per phase & 4 \\
\hline DC input voltage $\left(\mathrm{V}_{\text {in }}\right)$ & $100 \mathrm{~V}$ \\
\hline Switching frequency $\left(\mathrm{f}_{\mathrm{sw}}\right)$ & $50 \mathrm{~K} \mathrm{~Hz}$ \\
\hline fundamental frequency & $50 \mathrm{~Hz}$ \\
\hline Output Resistor (R) & $30 \Omega$ \\
\hline ALL Capacitors (C) & $10 \mu \mathrm{F}$ \\
\hline ALL inductors (L) & $10 \mathrm{mH}$
\end{tabular}

Table 1

The three-phase output voltage and currents are shown in Fig. 6 and Fig. 7 while the input DC current of the first PV module is shown in Fig. 8. Each SM generates two 
output voltages with opposite polarities, from a single input PV voltage as it is shown in Fig9.

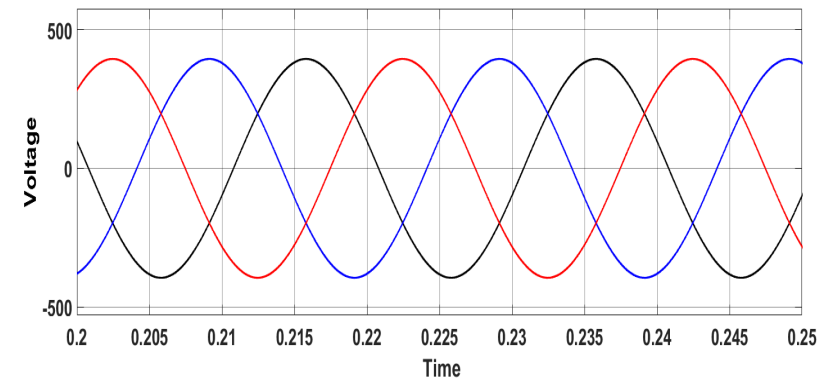

Figure 6 Three-phase voltages

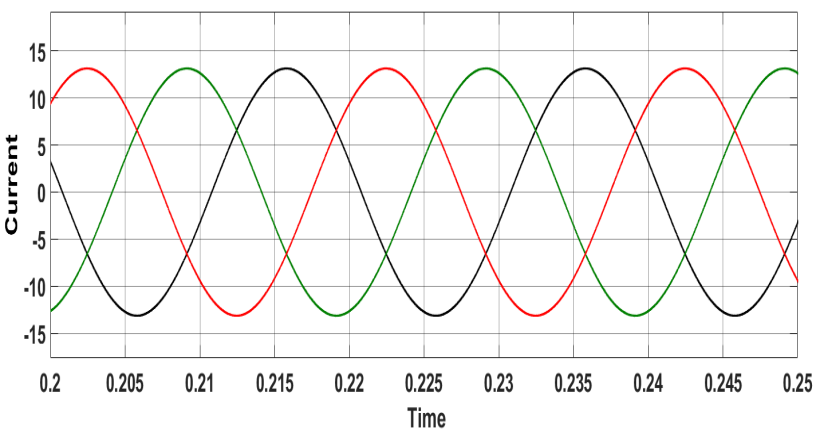

Figure 7 Three-phase currents

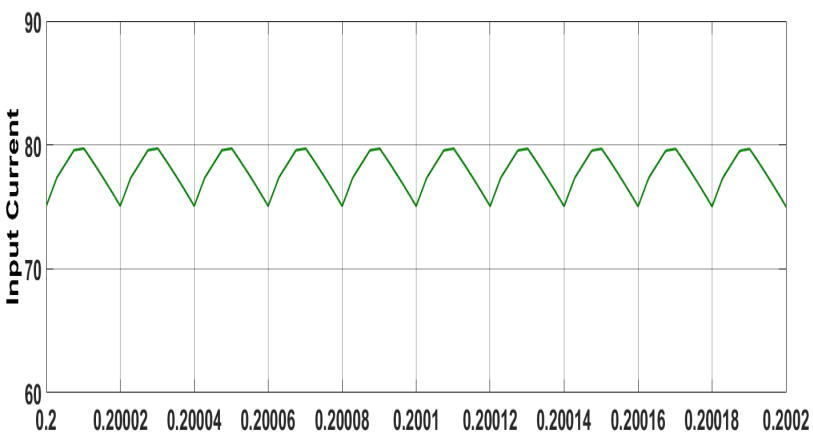

Figure 8 Input DC current
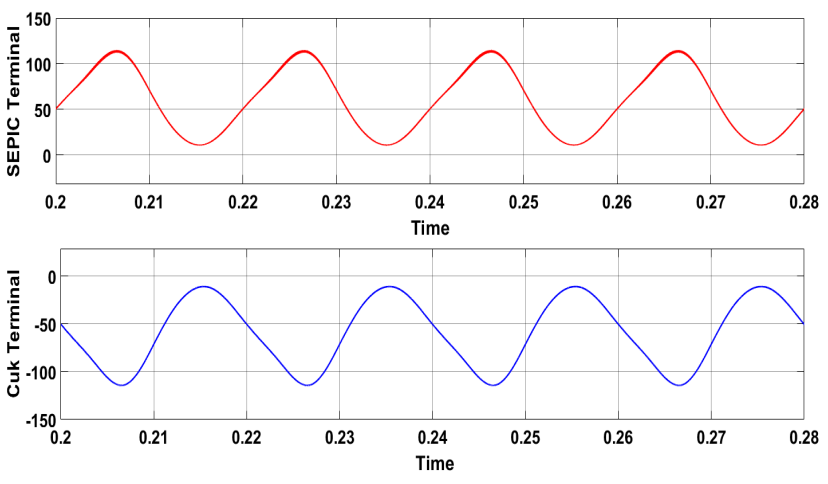

Figure 9 SM1 voltages

\section{Experimental validation}

Fig. 10 shows the experimental setup used in this work. The system is controlled by TMS32028335 DSP and uses the parameters in Table 2. Each SM will deliver
$250 \mathrm{~W}$ and hence the total power is $3 \mathrm{~kW}$. The input voltages of the modules are controlled by an external power supplies to mimic the PV modules.

\begin{tabular}{cc} 
Parameters & Value \\
\hline (SMs) per phase & 4 \\
\hline DC input voltage $\left(\mathrm{V}_{\text {in }}\right)$ & $200 \mathrm{~V}$ \\
\hline switching Frequency $\left(\mathrm{F}_{\mathrm{c}}\right)$ & $50 \mathrm{~K} \mathrm{~Hz}$ \\
\hline fundamental frequency & $50 \mathrm{~Hz}$ \\
\hline Output Resistor (R) & $22 \Omega$ \\
\hline ALL Capacitors (C) & $30 \mu \mathrm{F}$ \\
\hline ALL inductors (L) & $11 \mathrm{mH}$ \\
\hline Semiconductor switches & IRG4PC50FPbF \\
\hline Diodes & FFSH40120ADN \\
\hline
\end{tabular}

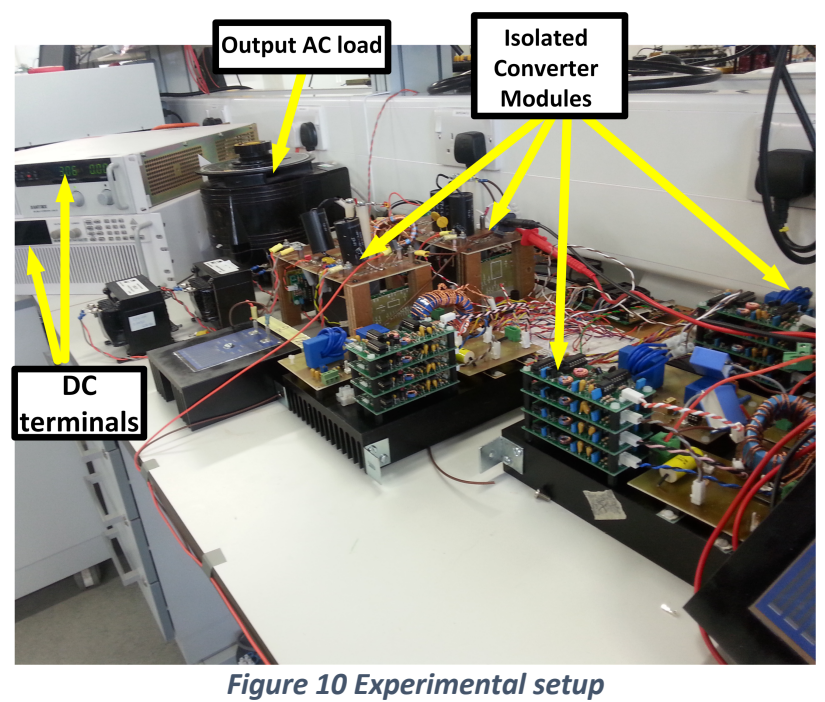

Fig. 11 shows the three-phase operation results when the system is generating $3 \mathrm{~kW}$ across the three $22 \Omega$ loads. Fig. 11a shows the phase a and Fig. 11b shows phase a current. The dual voltages of SMs in module 1 are shown in Fig. 11c. Fig. 11d shows the total input current of the 12 SMs together. To check the capability of the system in grid-connected mode, 1 phase is connected to the single-phase grid using a single-phase AC transformer and the voltage of common coupling is kept at $200 \mathrm{~V}$ (peak). Fig. 12 shows the ac output voltage, ac output current, SM1 output voltages and the total input current from the DC supply. 


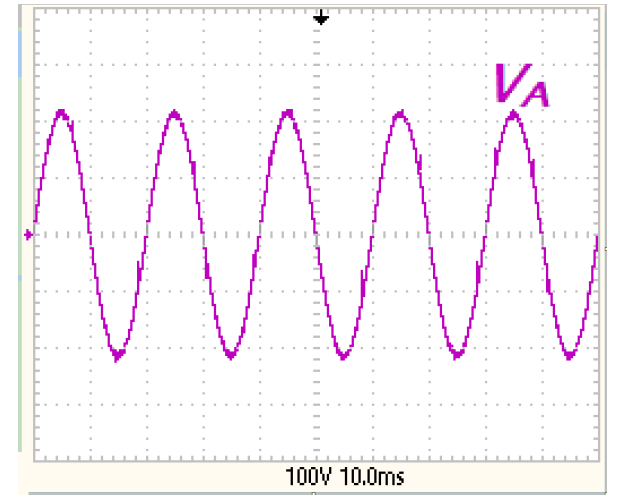

(a) Phase 1 voltage $100 \mathrm{~V} /$ div $-10 \mathrm{~ms} / \mathrm{div}$

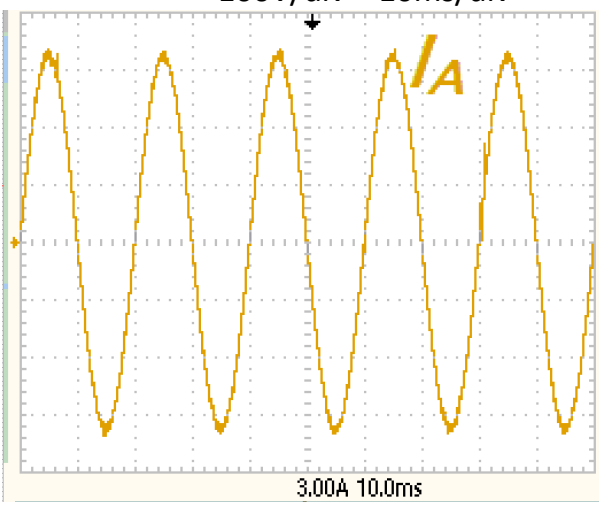

(b) Phase 1 current

$3 \mathrm{~A} / \mathrm{div}-10 \mathrm{~ms} / \mathrm{div}$

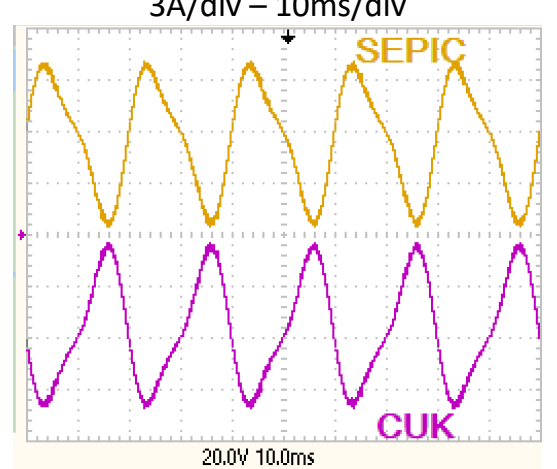

(c) SM1 voltages

$20 \mathrm{~V} /$ div $-10 \mathrm{~ms} /$ div

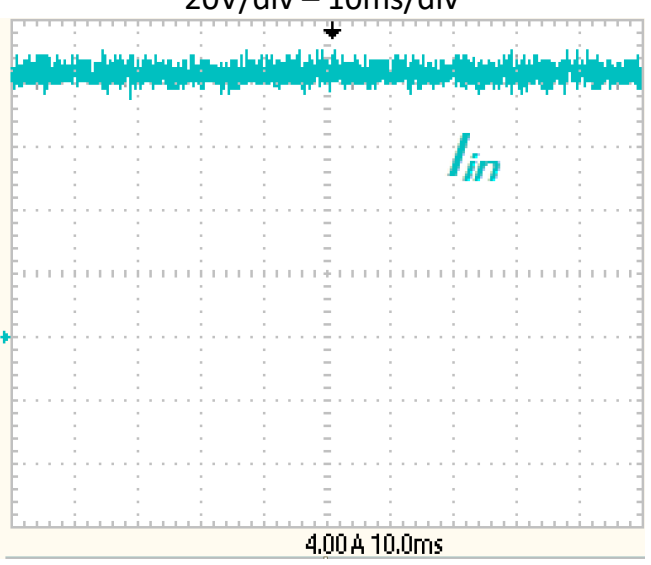

(d) Input DC current

$4 \mathrm{~A} / \mathrm{div}-10 \mathrm{~ms} / \mathrm{div}$

Figure 11 Three-phase operation

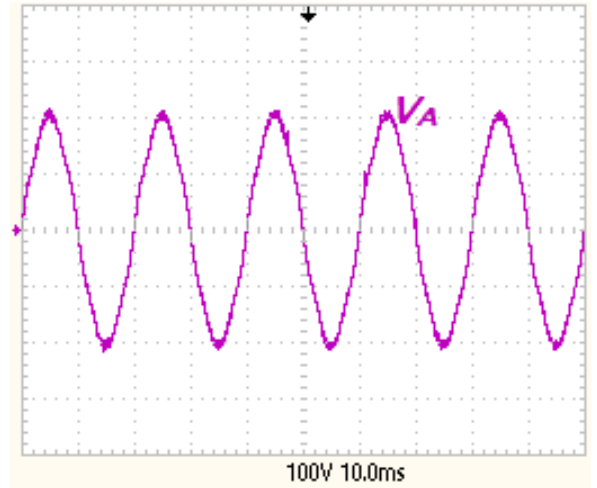

(a) Phase 1 voltage $100 \mathrm{~V} /$ div $-10 \mathrm{~ms} /$ div

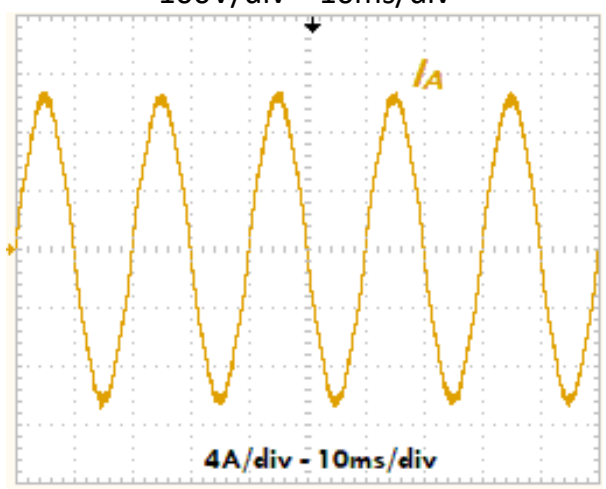

(b) Phase 1 current 4A/div - 10ms/div

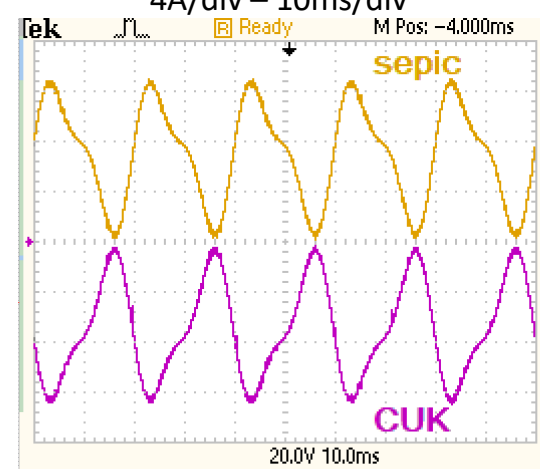

(c) SM1 voltages

20V/div-10ms/div

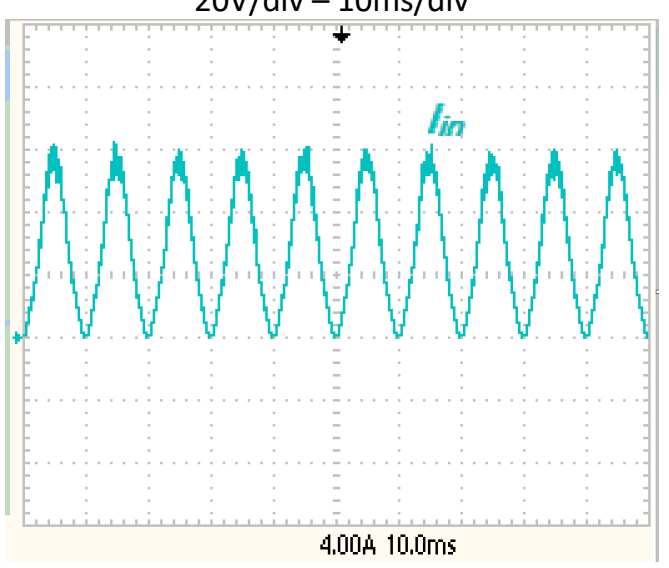

(d) Input DC current

$4 \mathrm{~A} / \mathrm{div}-10 \mathrm{~ms} / \mathrm{div}$

Figure 12 Single-phase operation 


\section{Conclusion}

The paper presents a new three-phase modular seriesconnected system for medium-voltage PV applications. The proposed inverter has attractive features that nominate it as a modern generation power inverter for large-scale PV system. This fact comes from its ability to: (i) operate as a current sourced converter with continuous input current, (ii) enable bidirectional power flow and galvanic isolation versions, (iii) provide a high level of modularity and scalability, and (iv) offer a flexible output voltage range which is necessary for the control schemes. Eventually, a control design strategy and an appropriate (Maximum Power Point Tracking) MPPT system will be presented in future publications.

\section{Acknowledgements}

Shaqra University is gratefully acknowledged, and the present research has been done within its support.

\section{References}

1. A. Whiteman, T. Rinke, J. Esparrago, and S. Elsayed, "Renewable Capacity Statistics 2016," Tech. Rep., 2016. [Online]. Available: www.irena.org

2. "Solar market report and membership directory," Solar Power Europe, 2016. [Online]. Available: https://zerohomebills.com/wpcontent/uploads/2016/04/2016-Solar-MarketReport.pdf, [Accessed: 19- Apr- 2020].

3. A. Darwish Badawy, " Current source dc-dc and dcac converters with continuous energy flow," Degree of Doctor of Philosophy, Department of Electronics and Electrical Engineering, University of Strathclyde, Glasgow, 2015.

4. M. Rabiul Islam, A. Mahfuz-Ur-Rahman, K. Muttaqi and D. Sutanto, "State-of-the-Art of the MediumVoltage Power Converter Technologies for Grid Integration of Solar Photovoltaic Power Plants", IEEE Transactions on Energy Conversion, vol. 34, no. 1, pp. 372-384, 2019.

5. A. Darwish and M. Elgenedy, "Current-Source Modular Medium-Voltage Grid-Connected System With High-Frequency Isolation for Photovoltaic Applications", IEEE Transactions on Energy Conversion, vol. 34, no. 1, pp. 255-266, 2019.

6. G. Petrone, G. Spagnuolo, R. Teodorescu, M. Veerachary and M. Vitelli, "Reliability Issues in Photovoltaic Power Processing Systems", IEEE Transactions on Industrial Electronics, vol. 55, no. 7, pp. 2569-2580, 2008.
7. M. Hannan, M. Lipu, P. Ker, R. Begum, V. Agelidis and F. Blaabjerg, "Power electronics contribution to renewable energy conversion addressing emission reduction: Applications, issues, and recommendations", Applied Energy, vol. 251, p. 113404, 2019.

8. A. Darwish, A. Massoud, D. Holliday, S. Ahmed and B. Williams, "Generation, performance evaluation and control design of single-phase differential-mode buck-boost current-source inverters," in IET Renewable Power Generation, vol. 10, no. 7, pp. 916927, 72016.

9. A. Darwish, A. Elserougi, A. Abdel-Khalik, S. Ahmed, A. Massoud, D. Holliday, and B. Williams, "A single-stage three-phase DC/AC inverter based on Cuk converter for PV application," in GCC Conference and Exhibition (GCC), 2013 7th IEEE, 2013, pp. 384-389

10. A. Darwish, S. Alotaibi, and M. A. Elgenedy. "Current-source Single-phase Module Integrated Inverters for PV Grid-connected Applications." IEEE Access (2020). 